\title{
ABOUT CALCULATION OF THE HANKEL TRANSFORM USING PRELIMINARY WAVELET TRANSFORM
}

\section{E. B. POSTNIKOV}

Received 15 August 2002 and in revised form 14 October 2002

The purpose of this paper is to present an algorithm for evaluating Hankel transform of the null and the first kind. The result is the exact analytical representation as the series of the Bessel and Struve functions multiplied by the wavelet coefficients of the input function. Numerical evaluation of the test function with known analytical Hankel transform illustrates the proposed algorithm.

The Hankel transform is a very useful instrument in a wide range of physical problems which have an axial symmetry [5]. The influence of the Laplacian on a function in a cylindrical coordinates is equal to the product of the squared parameter of the transformation and the transform of the function

$$
\begin{array}{r}
\left(\frac{d^{2}}{d r^{2}}+\frac{1}{r} \frac{d}{d r}\right) f(r) \longleftrightarrow-p^{2} F_{0}(p), \\
\left(\frac{d^{2}}{d r^{2}}+\frac{1}{r} \frac{d}{d r}-\frac{1}{r^{2}}\right) f(r) \longleftrightarrow-p^{2} F_{1}(p) .
\end{array}
$$

The Hankel transforms of the null $(n=0)$ and the first $(n=1)$ kind are represented as

$$
\begin{aligned}
& F_{n}(p)=\int_{0}^{\infty} f(r) J_{n}(p r) r d r \\
& f_{n}(p)=\int_{0}^{\infty} F(p) J_{n}(p r) p d p .
\end{aligned}
$$


Besides, those integrals like (2) are connected with the problems of geophysics and cosmology, for example, [6,8].

However, practical calculation of direct and inverse Hankel transform is connected with two problems. The first problem is based on the fact that not every transform in the real physical situation has analytical expression for result of inverse Hankel transform. The second one is the determination of functions as a set of their values for numerical calculations. Large bibliography on those issues can be found in [4]. The classical trapezoidal rule, Cotes rule, and other rules connected with the replacement of integrand by sequence of polynoms have high accuracy if integrand is a smooth function. But $f(r) J_{n}(p r) r$ (or $F_{p}(p) J_{n}(p r) p$ ) is a quick oscillating function if $r$ (or $p$ ) is large. There are two general methods of the effective calculation in this area. The first is the fast Hankel transform [7]. The specification of that method is transforming the function to the logarithmical space and fast Fourier transform in that space. This method needs a smoothing of the function in logs pace. The second method is based on the separation of the integrand into product of slowly varying component and a rapidly oscillating Bessel function [2]. But it needs the smoothness of the slow component for its approximation by low-order polynoms.

The goal of this paper is to apply wavelet transform with Haar bases to $(2)$.

The both direct and inverse transforms (2) are symmetric. Consider only one of them, for example, direct transform. Denote $f(r) r$ as $g(r)$. Then, the Hankel transform is

$$
F_{0,1}(p r)=\int_{0}^{\infty} g(r) J_{0,1}(p r) d r
$$

The expansion $g(r) \in L^{2}(R)$ into wavelet series with the Haar bases is (see [3])

$$
\begin{gathered}
g(r)=\sum_{k=0}^{\infty} c_{0 k} \varphi_{k}(r)+\sum_{j=0}^{\infty} \sum_{k=0}^{\infty} d_{j k} \psi_{j k}(r), \\
\varphi_{0 k}^{H}(r)=\varphi^{H}(r-k), \quad \psi_{j k}^{H}(r)=2^{j / 2} \psi^{H}\left(2^{j / 2} r-k\right), \\
\varphi^{H}(t)=\left\{\begin{array}{ll}
1, & t \in(0,1), \\
0, & t \notin(0,1),
\end{array} \psi^{H}(t)= \begin{cases}1, & t \in\left(0, \frac{1}{2}\right), \\
-1, & t \in\left(\frac{1}{2}, 0\right), \\
0, & t \notin(0,1) .\end{cases} \right.
\end{gathered}
$$


After substituting (4) into (3), one has

$$
\begin{aligned}
F_{0,1}(p)= & \sum_{k=0}^{\infty} c_{0 k} \int_{0}^{\infty} \varphi_{k}(r) J_{0,1}(p r) d r \\
& +\sum_{j=0}^{\infty} \sum_{k=0}^{\infty} d_{j k} \int_{0}^{\infty} \psi_{j k}(x) J_{0,1}(p r) d r
\end{aligned}
$$

Making use of integrals of [1], we have, as a result,

$$
\begin{aligned}
F_{0}(p)=\frac{1}{p}\left\{\sum _ { k \in Z } c _ { 0 k } \left[(k+1) J_{0}(p(k+1))-k J_{0}(p k)\right.\right. \\
\left.+\frac{\pi}{2}[(k+1) D(p(k+1))-k D(p k)]\right] \\
+\sum_{j=0}^{\infty} \sum_{k \in Z} d_{j k}\left[2\left(k+\frac{1}{2}\right) J_{0}\left(p\left(k+\frac{1}{2}\right)\right)-(k+1) J_{0}(p(k+1))\right. \\
-k J_{0}(p k)-\frac{\pi}{2}\left[2\left(k+\frac{1}{2}\right) D\left(p\left(k+\frac{1}{2}\right)\right)\right. \\
-(k+1) D(p(k+1))-k D(p k)]]\},
\end{aligned}
$$

$$
\begin{aligned}
F_{1}(p)=\frac{1}{p}\left\{\sum_{k \in Z} c_{0 k}\left[J_{0}(p k)-J_{0}(p(k+1))\right]\right. \\
+\sum_{j=0}^{\infty} \sum_{k \in Z} d_{j k}\left[2 J_{0}\left(p\left(k+\frac{1}{2}\right) 2^{-j}\right)\right. \\
\left.\left.\quad-J_{0}\left(p(k+1) 2^{-j}\right)-J_{0}\left(p k 2^{-j}\right)\right]\right\},
\end{aligned}
$$

where $D(\xi)=H_{0}(\xi) J_{1}(\xi)-H_{1}(\xi) J_{0}(\xi)$ and $H_{0,1}$ is a Struve function of the null and the first kind.

The most sufficient result is that (8) and (9) are exact. They can be used in any analytical expressions. Especially it is useful for Hankel transform of the first kind because (9) contains only a combination of Bessel functions, and one can use their properties such as orthogonality, 
known locality of the zeros, and extremums. The coefficients $c_{0 k}$ means average value of $g(r)$ at the range $[k, k+1]$ is

$$
c_{0 k}=\int_{k}^{k+1} g(r) d r
$$

The detail coefficients are

$$
d_{j k}=2^{j / 2}\left\{\int_{2^{-j k}}^{2^{-j}(k+1 / 2)} g(r) d r-\int_{2^{-j}(k+1 / 2)}^{2^{-j}(k+1)} g(r) d r\right\} .
$$

Formulas (8) and (9) allow us to get a full analytical solution if the integrals above have close form solution. In the opposite case, the solution must be numerical but this method provides an effective algorithm for that. It is obvious that $d_{j k}$ decrease very quickly if $g(r)$ is a smooth function. One can practically use $d_{j k}>\varepsilon$, where $\varepsilon$ is small. The largest detail coefficients are concentrated around steps, sharp vertices, and discontinues of $g(r)$; and one can appropriate that they are equal to zero in other areas.

Consider, for example, a function with known analytical Hankel transform

$$
\int_{0}^{\infty} e^{-a^{2} r^{2}} r J_{1}(p r) r d r=\frac{p}{4 a^{4}} e^{-p^{2} / 4 a^{2}}
$$

The approximation and detail coefficients may be calculated analytically in a closed form

$$
\begin{aligned}
c_{0 k}= & \left.\frac{\sqrt{\pi} \operatorname{erf}(r)-2 a r e^{-a^{2} r^{2}}}{4 a^{3}}\right|_{k} ^{(k+1)}, \\
d_{j k}= & \left.2^{j / 2} \frac{\sqrt{\pi} \operatorname{erf}(r)-2 a r e^{-a^{2} r^{2}}}{4 a^{3}}\right|_{2^{-j} k} ^{2^{-j}(k+1 / 2)} \\
& -\left.2^{j / 2} \frac{\sqrt{\pi} \operatorname{erf}(r)-2 a r e^{-a^{2} r^{2}}}{4 a^{3}}\right|_{2^{-j}(k+1 / 2)} ^{2^{-j}(k+1)} .
\end{aligned}
$$

Thus (9), with the coefficients (13), is the exact representation of the Hankel transform. Consider the approximate solution. Suppose that the function (12) is known only in the segment $[0, h]$. Then there is the series, instead of (4),

$$
g(r)=c_{0 k} \varphi_{0}(r)+\sum_{j=0}^{J} \sum_{k=0}^{2^{J}-1} d_{j k} \psi_{j k}(r)
$$




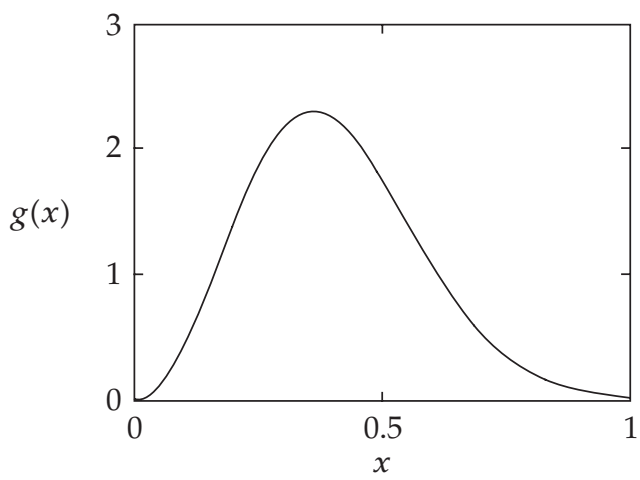

(a)

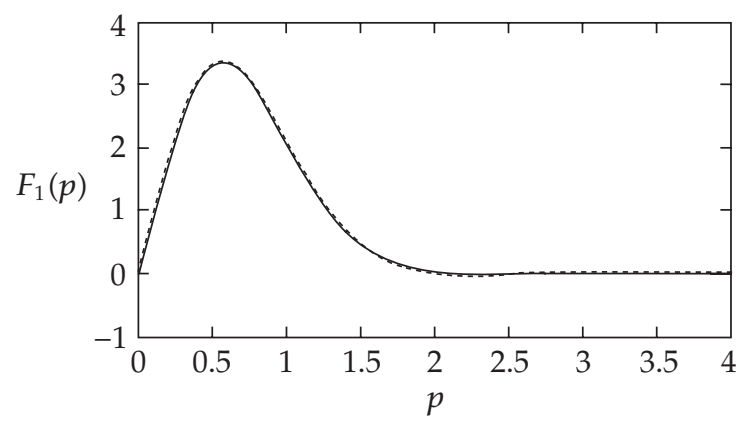

(b)

FIGURE 1. (a) Original function and (b) Hankel transform.

If $J \rightarrow \infty$, then (14) is exact for this truncated function. In practice, one uses only small $J$, up to $3-4$. For example, we can see the original function (12) (the replacement $r$ to $x=r / h$ is used) and the transform in Figure 1. One can see that the exact transform (solid line) and the transform at level $J=3$ (dotted line) coincide in this figure. The absolute errors between the exact transform and the approximate transform at the levels $J=2$ (solid line), $J=3$ (dashed line), and $J=4$ (dotted line) are represented in Figure 2a. It is oblivious that the error is small in comparison with the values of the $F_{1}(p)$. The absolute error at the level $J=3$ in a wide range of $p$ is plotted in Figure 2a. One can see that this error has quasiperiodic oscillations because the function is truncated. But they 
324 About calculation of the Hankel transform

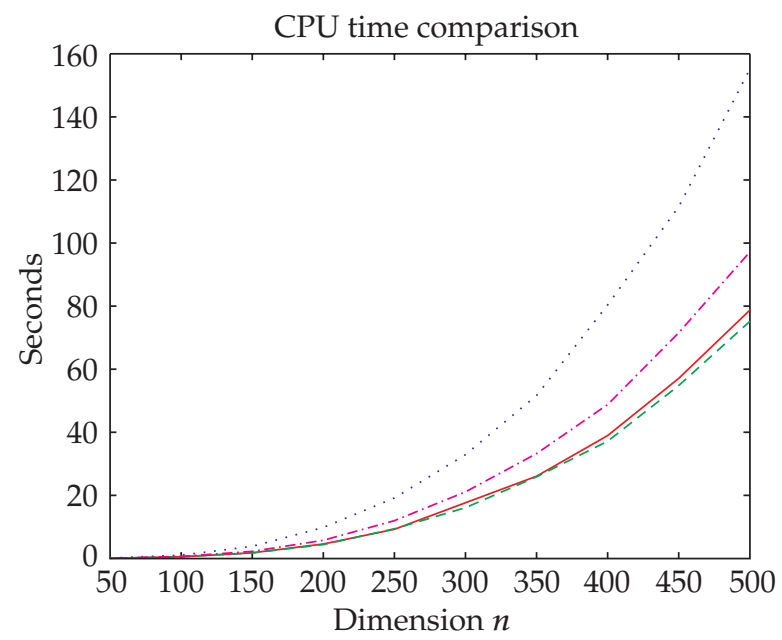

Matlab: lyap $\quad--2$-solve

- 1-solve -.- E-solve

(a)

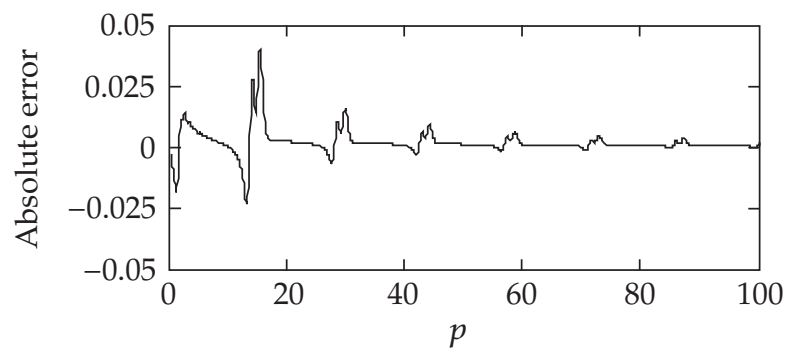

(b)

FIgURE 2. Transform's error.

decrease with the growth of $p$ (and $J$ ) when oscillations in classical fast Hankel transform [6] increase.

\section{References}

[1] M. Abramowitz and I. A. Stegun, Handbook of Mathematical Functions with Formulas, Graphs, and Mathematical Tables, National Bureau of Standards Applied Mathematics Series, vol. 55, U.S. Government Printing Office, Washington, D.C., 1964. 
[2] R. Barakat and E. Parshall, Numerical evaluation of the zero-order Hankel transform using Filon quadrature philosophy, Appl. Math. Lett. 9 (1996), no. 5, 21-26.

[3] C. K. Chui, An Introduction to Wavelets, Wavelet Analysis and Its Applications, vol. 1, Academic Press, Massachusetts, 1992.

[4] D. W. Lozier and F. W. J. Olver, Numerical evaluation of special functions, Mathematics of Computation 1943-1993: A Half-Century of Computational Mathematics (Vancouver, BC, 1993) (W. Gautschi, ed.), Proc. Sympos. Appl. Math., vol. 48, American Mathematical Society, Rhode Island, 1994, pp. 79-125.

[5] J. Mathews and R. L. Walker, Mathematical Methods of Physics, W. A. Benjamin, New York, 1964.

[6] Hamilton A. J. S., Uncorrelated modes of the nonlinear power spectrum, Monthly Notices Roy. Astronom. Soc. (2000), no. 312, 257-284.

[7] A. E. Siegman, Quasi fast Hankel transform, Optics Lett. 1 (1977), 13-15.

[8] J. Zhao, W. W. M. Dai, S. Kapur, and D. E. Long, Efficient three-dimensional extraction based on static and full-wave layered Green's functions, Proceedings of the 35th Conference on Design Automation (San Francisco, Calif, 1998), ACM Press, New York, 1998, pp. 224-229.

E. B. Postnikov: Theoretical Physics Department, Kursk State Pedagogical University, Radischeva st. 33, Kursk 305000, Russia

E-mail address: postnicov@mail.ru 


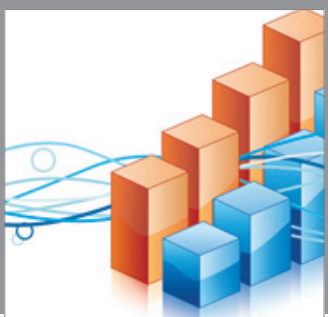

Advances in

Operations Research

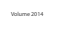

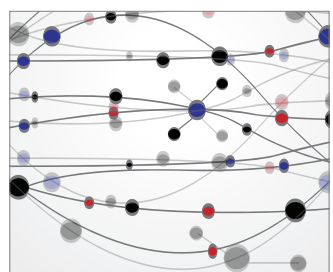

\section{The Scientific} World Journal
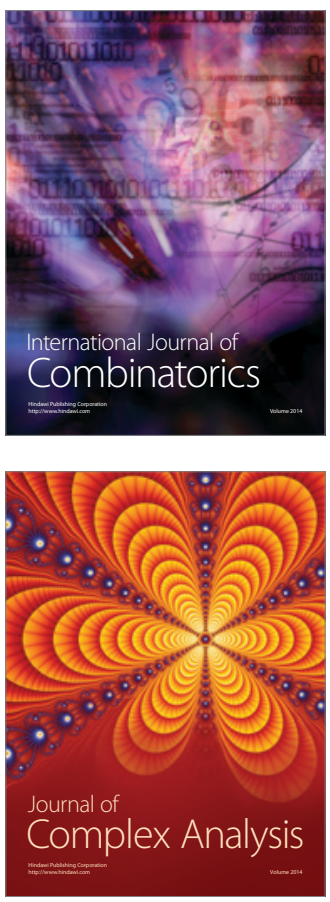

International Journal of

Mathematics and

Mathematical

Sciences
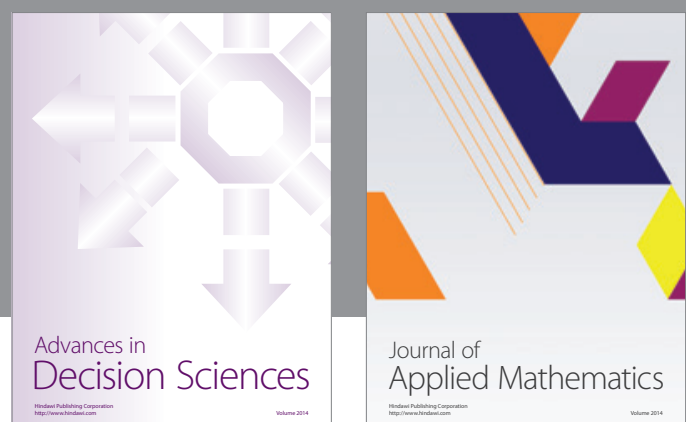

Journal of

Applied Mathematics
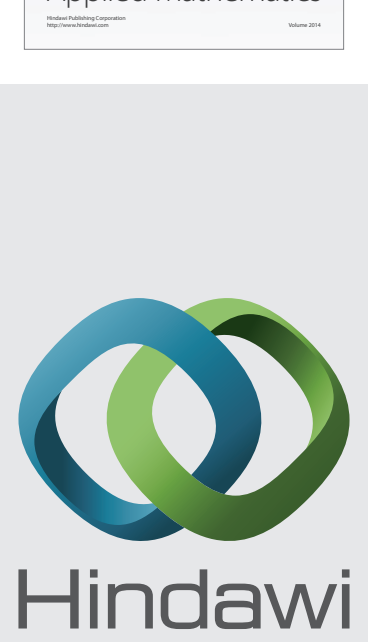

Submit your manuscripts at http://www.hindawi.com
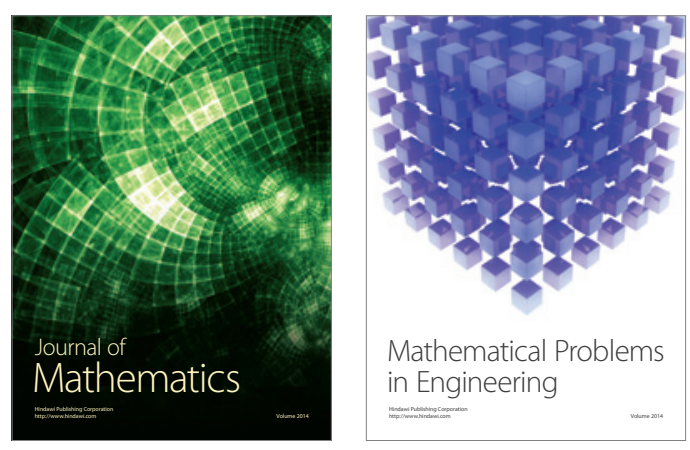

Mathematical Problems in Engineering
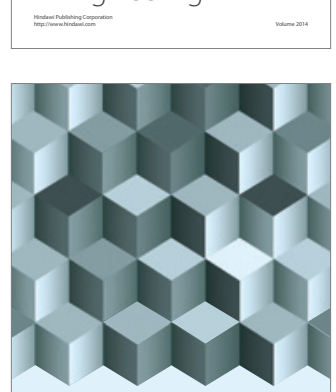

Journal of

Function Spaces
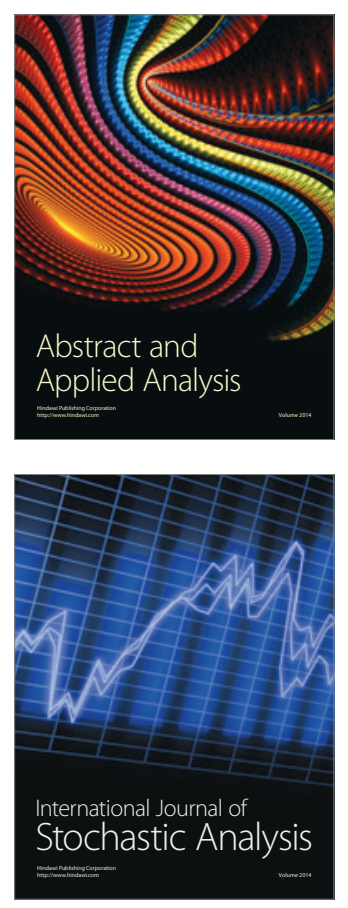

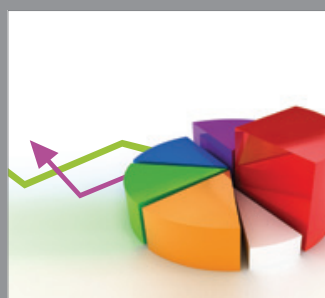

ournal of

Probability and Statistics

Promensencen
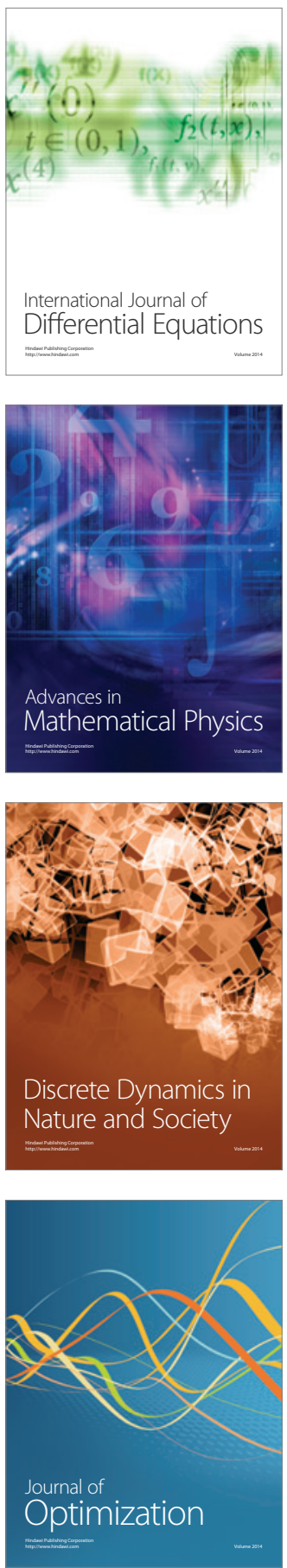